\title{
Posterior basal segments atelectasis in descending necrotizing mediastinitis
}

\author{
Benito Vargas-Ábrego*, Francisco P. Navarro-Reynoso, Raul J. Cicero-Sabido, and Leon Green-Schneweiss \\ Department of Thoracic Surgery at Hospital General de México "Dr. Eduardo Liceaga," Mexico City, Mexico
}

\begin{abstract}
Background: We have observed that patients with descending necrotizing mediastinitis show bilateral lower lobes atelectasis before pleural effusion that finally forms empyema. Successful treatment of mediastinitis emphasizes optimal early drainage of neck, mediastinum, and antibiotic therapy. Methods: Exploratory study to determine the presence of segmental atelectasis as incipient sign of mediastinitis. We studied patients from August 2018 to July 2019 with tomographies. Results: We evaluated 25 patients operated due to Mediastinitis at our hospital, from August 2018 to July 2019. Ten (40\%) were females and 15 (60\%) were male patients. Twenty-one (84\%) patients showed atelectasis and 4 (16\%) did not have atelectasis. Of the patients that showed atelectasis, 17 (81\%) were bilateral, 2 (9.5\%) were right sided, and 2 (9.5\%) were left sided. We also analyzed the occurrence of pleural effusion in this group. Fifteen (60\%) patients had pleural effusion and $10(40 \%)$ did not. Of the total patients with pleural effusion 11 (73.3\%) were bilateral, $3(20 \%)$ of the right side, and 1 (6.6\%) of the left side. Conclusions: Atelectasis is a sign that we can find in most patients that will develop empyema and we assume that it is associated with lymph nodes inflammation, but it is necessary to develop other protocols to confirm that hilar lymph congestion is the cause of atelectasis. As atelectasis of lower lobes is a sign of imminent sepsis, we always decide to do mediastinal drainage when we find them associated with neck abscess. We are proposing a modification of the endo classification to decide surgery.
\end{abstract}

Key words: Atelectasis. Mediastinitis. Mediastinal drainage.

\section{Introduction}

Atelectasis means imperfect expansion, and the word is used to describe incomplete expansion of a lung or lung tissue ${ }^{1}$. René Laennec was the first to describe atelectasis as a finding during an autopsy in $1819^{2}$. Asrar shows the association of descending necrotizing mediastinitis with left lower lobe atelectasis and hilar lymph node in a single patient. He mentions there that lung collapse can be due to external pressure of the bronchus owing to hilar lymphadenopathy ${ }^{3}$.

Kornblum and Osmond defined Mediastinitis since 1934 as inflammation of the loose mediastinal areolar tissue and its blood lymph and nerve supply ${ }^{4}$. The most known classification of mediastinitis is the one that Estrera and cols have described since $1983^{5}$. Another classification that we use is the one of Endo et al. that describes situations where infection has spread to posterior mediastinum, particularly when it reaches in the level of the carina (descending necrotizing mediastinitis-type I), may not always require aggressive mediastinal drainage. In comparison, diffuse descending necrotizing mediastinitis-Type IIB demands complete (Table 1$)^{6}$. Corsten demonstrated that successful drainage of patients with descending necrotizing mediastinitis emphasizes

\section{Correspondence:}

*Benito Vargas-Ábrego

E-mail: dr.benito.vargas@gmail.com

0185-1063/@ 2020 Sociedad Médica del Hospital General de Mexico. Published by Permanyer. This is an open access article under the CC BY-
NC-ND license (http://creativecommons.org/licenses/by-nc-nd/4.0/).
Date of reception: 09-09-2019

Date of acceptance: 08-09-2020
Available online: 19-04-2021

Rev Med Hosp Gen Mex. 2021;84(2):55-58 www.hospitalgeneral.mx NC-ND license (http://creativecommons.org/licenses/by-nc-nd/4.0/). 
Table 1. Endo's guideline of surgical management based on diffusion of descending necrotizing mediastinitis

\begin{tabular}{|l|l|l|}
\hline Type & $\begin{array}{l}\text { Zone of the } \\
\text { mediastinum infected }\end{array}$ & $\begin{array}{l}\text { Recommended surgical } \\
\text { procedure }\end{array}$ \\
\hline I & $\begin{array}{l}\text { Localized to the upper } \\
\text { mediastinal space } \\
\text { above the carina }\end{array}$ & $\begin{array}{l}\text { Transcervical mediastinal } \\
\text { drainage }\end{array}$ \\
\hline IIA & $\begin{array}{l}\text { Extended to the lower } \\
\text { anterior mediastinum } \\
\text { below the carina }\end{array}$ & $\begin{array}{l}\text { Transcervicotomy and } \\
\text { anterior mediastinal drainage } \\
\text { through a subxiphoidal } \\
\text { incision }\end{array}$ \\
\hline IIB & $\begin{array}{l}\text { Extended to both } \\
\text { anterior and posterior } \\
\text { lower mediastinum } \\
\text { below the carina }\end{array}$ & $\begin{array}{l}\text { Posterior mediastinal } \\
\text { drainage through a right } \\
\text { standard thoracotomy } \\
\text { followed by left minimal } \\
\text { thoracotomy }\end{array}$ \\
\hline
\end{tabular}

the importance of optimal early drainage of both neck and mediastinum and prolonged antibiotic therapy?

Decision of thoracotomy in patients with deep neck abscess and no classic signs of mediastinitis in a tomography, such as mediastinal widening, air in the mediastinum, air in the connective tissue of the neck, pleural effusion or hydropneumothorax, is not easy. Controversies could exist about the existence of the diagnosis of mediastinitis or the only presence of a deep neck abscess. We have observed that most patients with deep neck abscess course with posterior basal segments, bilateral, or unilateral atelectasis associated with or without pleural effusion. We have seen patients with the previous thoracotomy that does not develop pleural effusion, making an even harder decision to make. We consider that some of the first tomographic signs to lead to the decision to drain the mediastinum are the presence of posterior basal segments atelectasis. We consider that it only takes some days to develop pleural effusion and empyema since the cause is the descent of the infection through the mediastinal connective tissue and lymph nodes leading to high mortality reported in the literature.

\section{Methods}

Observational study to determine the presence of atelectasis in a group of patients operated with the diagnosis of descending necrotizing mediastinitis at the Hospital General de México "Dr. Eduardo Liceaga." We registered all the patients operated in 1 year with the diagnosis of mediastinitis in a period of 12 months. Tomography was necessary to include the patients in the study, available at our hospital at the PACS Carestream software.
We used descriptive statistics to analyze the information obtained in the group of patients that we studied. The variables studied are described in percentages.

\section{Results}

We evaluated 25 patients that we operated to drain the mediastinum for the diagnosis of mediastinitis, following the Estrera and Endo criteria, at the Hospital General de México "Dr. Eduardo Liceaga," from August 12018 to July 31 2019. Ten (40\%) were female patients and $15(60 \%)$ were male patients. Twenty-one (84\%) patients showed atelectasis and $4(16 \%)$ did not have atelectasis. Of the patients that showed atelectasis, $17(81 \%)$ were bilateral, $2(9.5 \%)$ were right sided, and $2(9.5 \%)$ were left sided. We also analyzed the occurrence of pleural effusion in this group. Fifteen $(60 \%)$ patients had pleural effusion and $10(40 \%)$ did not. Of the total patients with pleural effusion 11 $(73.3 \%)$ were bilateral, $3(20 \%)$ of the right side, and $1(6.6 \%)$ of the left side (Figs. 1-3).

\section{Discussion}

From August 2018 to July 2019, we studied 25 patients with the diagnosis of descending necrotizing mediastinitis. About $80 \%$ of them had atelectasis and 20 did not. About $60 \%$ of the patients had pleural effusion and $40 \%$ did not. About $60 \%$ were males and $40 \%$ were females.

A few studies mention the presence of atelectasis when the diagnosis is mediastinitis such as the one of Asrar $^{3}$ Haz clic o pulse aquí para escribir texto.that proposes as a cause of atelectasis the inflammation of the hilar lymphadenopathy due to infection. At our study, we found a patient with a couple of right hilar nodes affected by inflammation that showed atelectasis and pleural effusion only at the right side, without any of both findings in the left side.

We have observed that most patients with deep neck abscess develop basal posterior segments, bilateral or unilateral atelectasis associated or not to pleural effusion. We have seen patients with previous thoracotomy that does not develop pleural effusion, making an even harder decision to make. We consider that some of the first tomographical signs to lead to the decision to drain the mediastinum are the presence of posterior basal segments atelectasis. We consider that it only takes some days to develop pleural effusion and empyema since the cause is the descent of the infection through the mediastinal connective tissue and lymph nodes leading to the high mortality reported in the literature. 


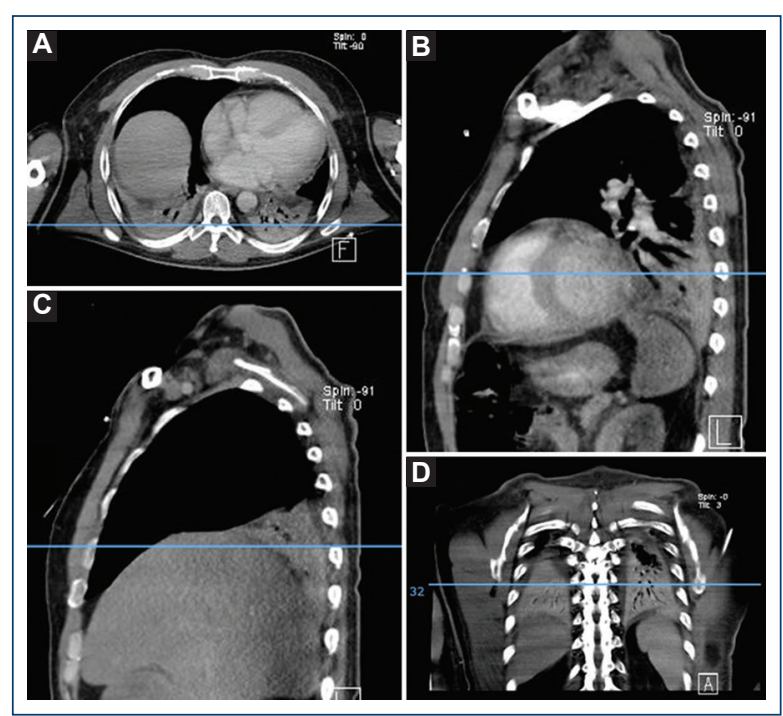

Figure 1. Patient with basal posterior segments atelectasis showing no pleural effusion. A: axial plane. B: sagittal plane left hemithorax. C: sagittal plane right hemithorax. D: coronal plane.

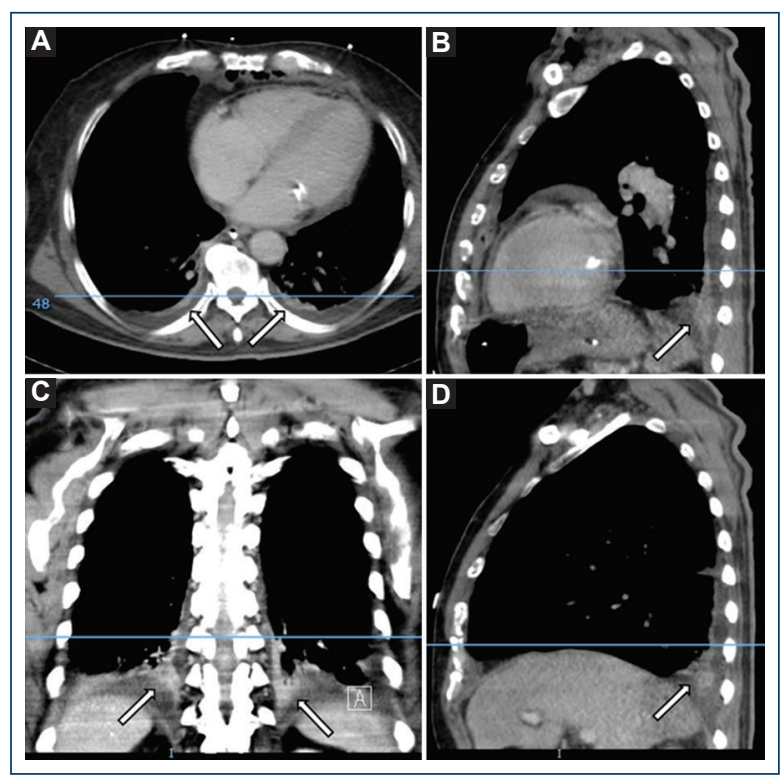

Figure 2. Patient with basal posterior segments atelectasis showing pleural effusion. A: axial plane. B: sagittal plane left hemithorax. C: coronal plane. D: sagittal plane right hemithorax.

Further studies are necessary to find out the etiology of atelectasis. For instance, it is necessary to do a lung biopsy of the collapsed lung and an affected lymph node, to request a pathologist to look for the presence of lymph fluid in the alveolar space. This biopsy could support the idea of mediastinal and hilar lymph nodes

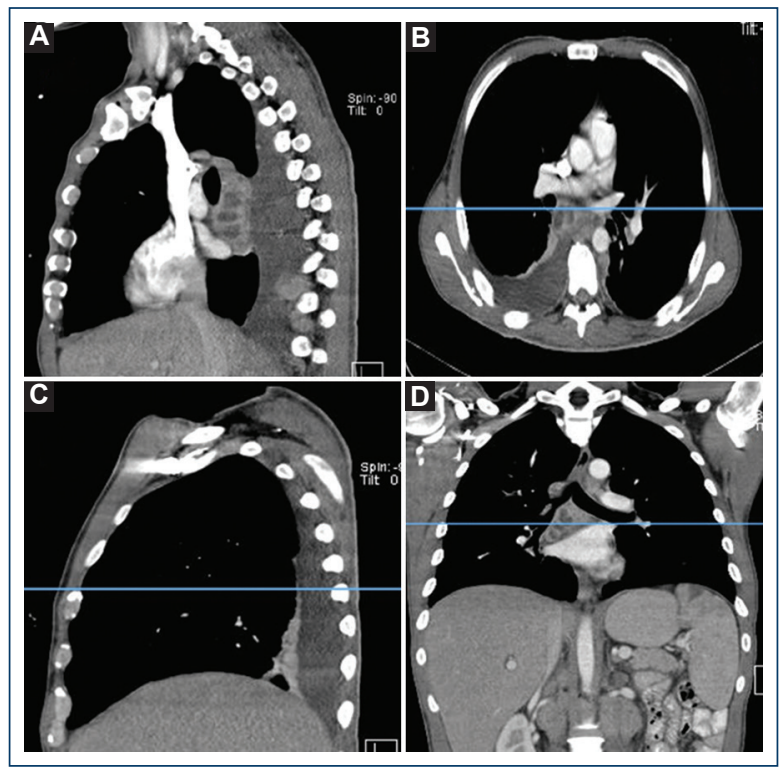

Figure 3. Patient with basal posterior segments atelectasis showing right basal posterior segment atelectasis, right pleural effusion, and right lymph nodes abscess suggesting lymph inflammation as a cause of atelectasis. A: sagittal plane left hemithorax. B: axial plane. C: sagittal plane right hemithorax. D: coronal plane.

blockage as a cause of atelectasis. Other type of studies is necessary to describe indication of thoracotomy with the only presence of atelectasis without pleural effusion. A new classification of mediastinitis is necessary to determine the exact indication of thoracotomy since endo's classification ${ }^{6} \mathrm{Haz}$ clic 0 pulse aquí para escribir texto.demands thoracotomy to all the patients that have affection below and posterior of the carina. At our hospital, we have a large data base of more than 1800 cases of mediastinitis to analyze every aspect of the disease and we can do a protocol to form the mentioned new proposal of classification ${ }^{8}$. Further information is necessary to know, if antibiotics are needed alone to avoid surgery in this group of patients.

We have not found an explanation of the etiology of atelectasis seen in patients with Mediastinitis since the two common causes are obstruction and compression. Some patients have atelectasis without pleural effusion and no evidence of obstruction. We did not find patients with pleural effusion without atelectasis.

\section{Conclusions}

Atelectasis is a sign that we can find in most patients that will develop empyema and we assume that it is 
associated with lymph nodes inflammation, but it is necessary to develop other protocols to confirm that hilar lymph congestion is the cause of atelectasis. As atelectasis of lower lobes is a sign of imminent sepsis, we always decide to do mediastinal drainage when we find them associated with neck abscess. We are proposing a modification of the endo classification to decide surgery.

\section{Conflict of interest}

The authors affirm that they have no conflicts of interest.

\section{Ethical disclosures}

Protection of human and animal subjects. The authors declare that no experiments were performed on humans or animals for this study.

Confidentiality of data. The authors declare that they have followed the protocols of their work center on the publication of patient data.
Right to privacy and informed consent. The authors have obtained the written informed consent of the patients or subjects mentioned in the article. The corresponding author is in possession of this document.

\section{References}

1. Carlsen KH, Crowley S, Smevik B. 70-Atelectasis. In: Kendig's Disorders of the Respiratory Tract in Children. Amsterdam: Elsevier Inc.; 2019. p. 1027-33.e1.

2. Laennec R. A Treatise on the Diseases of the Chest (De L'auscultation Médiate); 1819. Available from: http://www.books.google.com.

3. Asrar L, Dlo M, Mohammad R, Khan S. Segmental lower lobe collapse of the left lung associated with mediastinitis secondary to parapharyngeal abscess and quinsy. J Otolaryngol. 2004;33:260-3.

4. Kornblum K, Osmond L. Mediastinitis. Am J Roentgenol. 1934;32: 23-42.

5. Estrera A, Landay M,Grisham J, Sinn D, Platt M. Descending necrotizing mediastinitis. Surg Gynecol Obstet. 1983;157:545-52.

6. Endo S, Furayama F, Hasegawa T. Guideline of surgical management based on diffusion of descending necrotizing mediastinitis. Jpn J Thorac Cardiovasc Surgvol. 1999;47:14-9.

7. Corsten MJ, Shamji FM, Odell PF, Frederico JA, Laframboise GG, Reid KR, et al. Optimal treatment of descending necrotising mediastinitis. Thorax. 1997;52:702-8.

8. Navarro-Reynoso FP, Vargas-Abrego B, Pérez-Romo A, Cicero-Sabido R. Mediastinitis, a model of care. Experience in the general hospital of Mexico over 34 years (1982-2016). Rev Méd Hosp Gen Méx. 2017;80:22-32. 